\title{
AD Robotics - 3D Printer Enclosure
}

\section{Emily Stachowicz under the mentorship of Noah Curfman}

\section{Working to Prevent Failed 3D Prints}

Many of the clever minds at FNAL cannot wait on necessary parts to arrive. This immediate need of products leads to an increase in use of 3D printed objects. While 3D printers can provide almost immediate products, failed prints can cause even longer setbacks than having to wait on shipping with ordered parts. Prints affected by uneven temperature, air current, and/or debris in the air result in warping and other general failures.

\section{The Warping Issue}

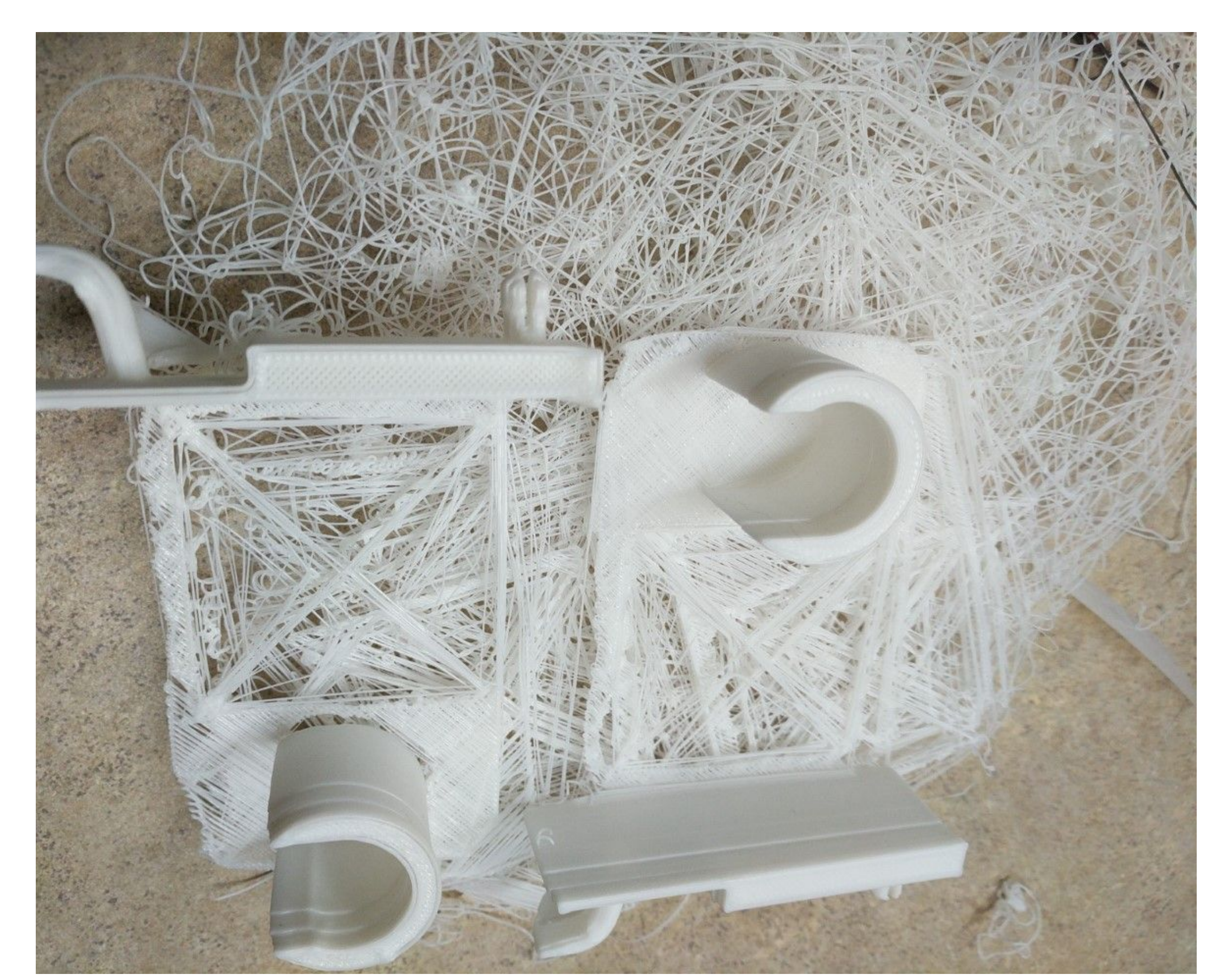

An example of a failed 3D print. Due to improper temperature maintenance, the print lifted from the print bed, ruining the needed component.

3D printed parts can fail for a variety of reasons. Temperature is an important factor in 3D printing. The layers of the print must set and cool at the same temperature for the finest results. Interference of airflow can cause warping due to premature curing of the 3D printed filament. Warping results in uneven layers, altering the rest of the print to be out of balance.

In addition to this, wind from passing by objects can cause the extruded filament to shift, producing inaccurate, uneven prints. Finding a way to prevent air current from interfering with anything on the print bed would prevent this issue from occurring.

Debris also plays a part in disfigured prints. Debris cannot be avoided without intervention and introduces further issues. Dust and other foreign particles in the air can eventually settle in unfavorable areas, such as on the in progress print. The addition of dust particles can cause further warping.

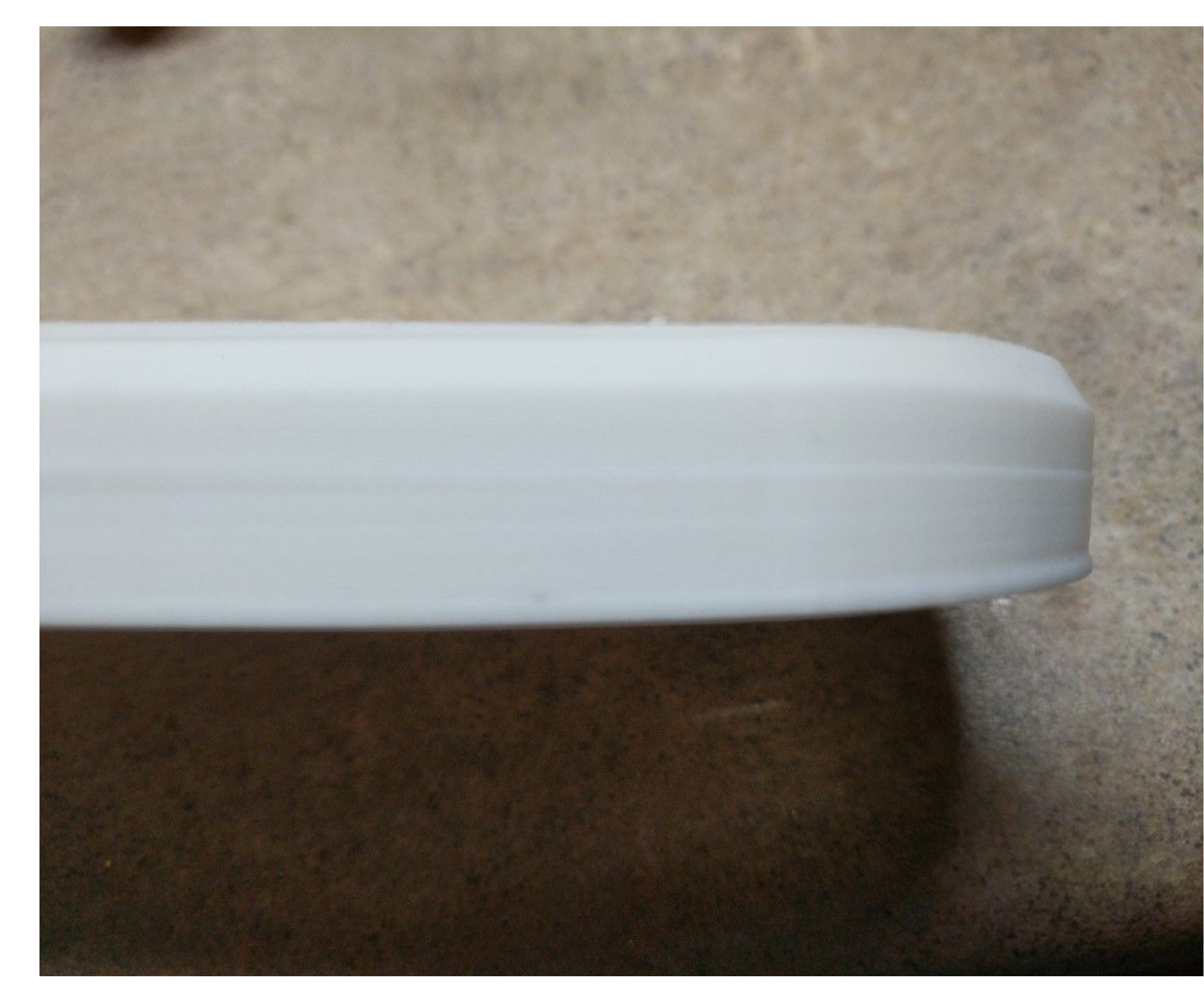

An example of a failed 3D print for a robotic arm. Print bed temperature was not maintained, causing for the print to warp upwards.

\section{The Effect on Fermi National Accelerator Laboratory}

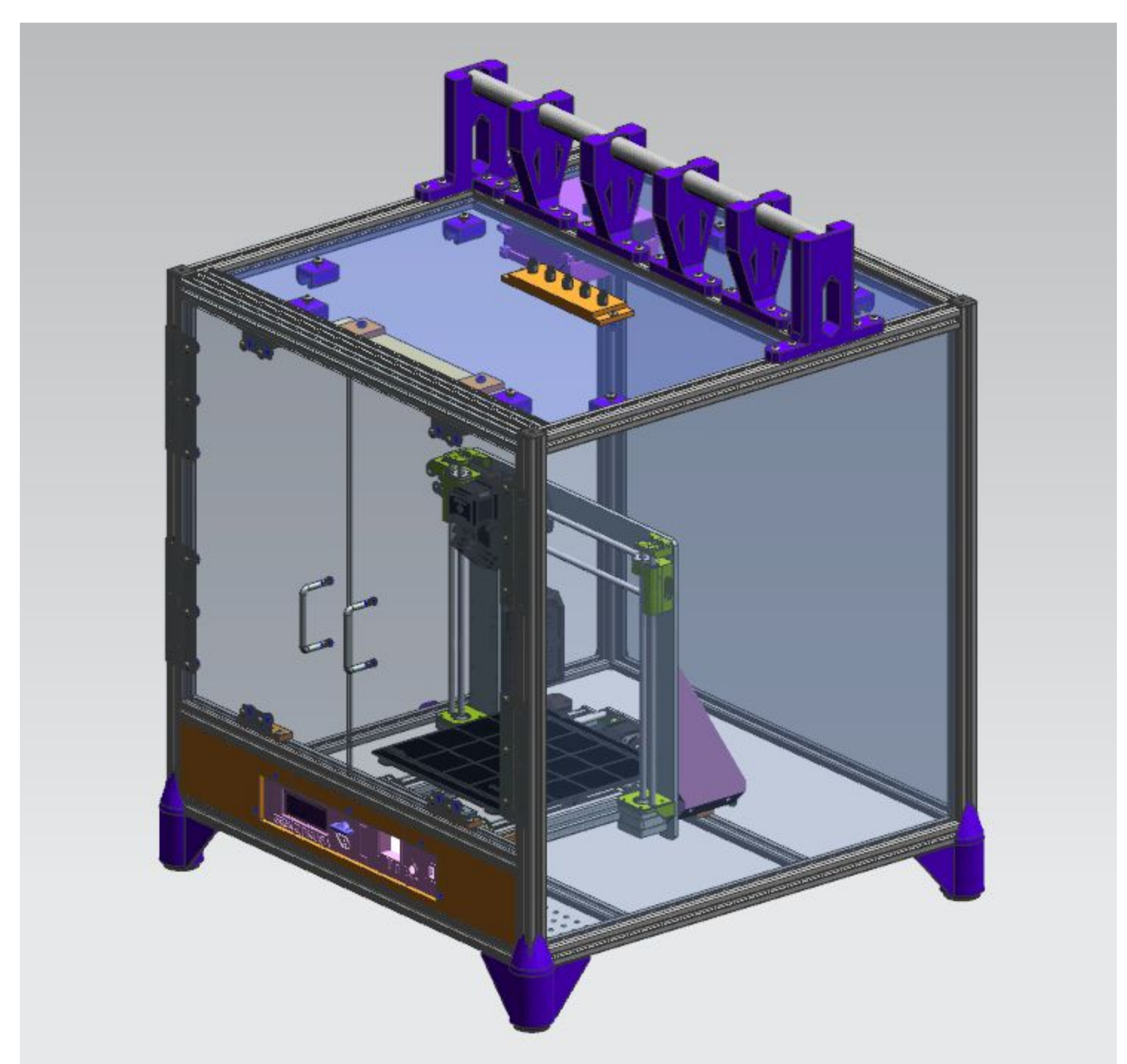

In this image, the final 3D model of the enclosure is shown, utilizing a combination of the two interns' designs and hard work.

Warping introduces unexpected structural changes to a print. Changing the structure of a print decreases overall product stability, making it unusable for it's originally intended purpose. When these products are designed, they are engineered to meet certain requirements, sometimes needing simulations to accurately assess the structural integrity.

Failed prints can also mislead researchers and engineers alike to think that their design itself is the problem, when in reality, it is unforeseen print issues. Ensuring stability of a print can make or break a project. The solution to this comes in the form of the 3D printer enclosure.

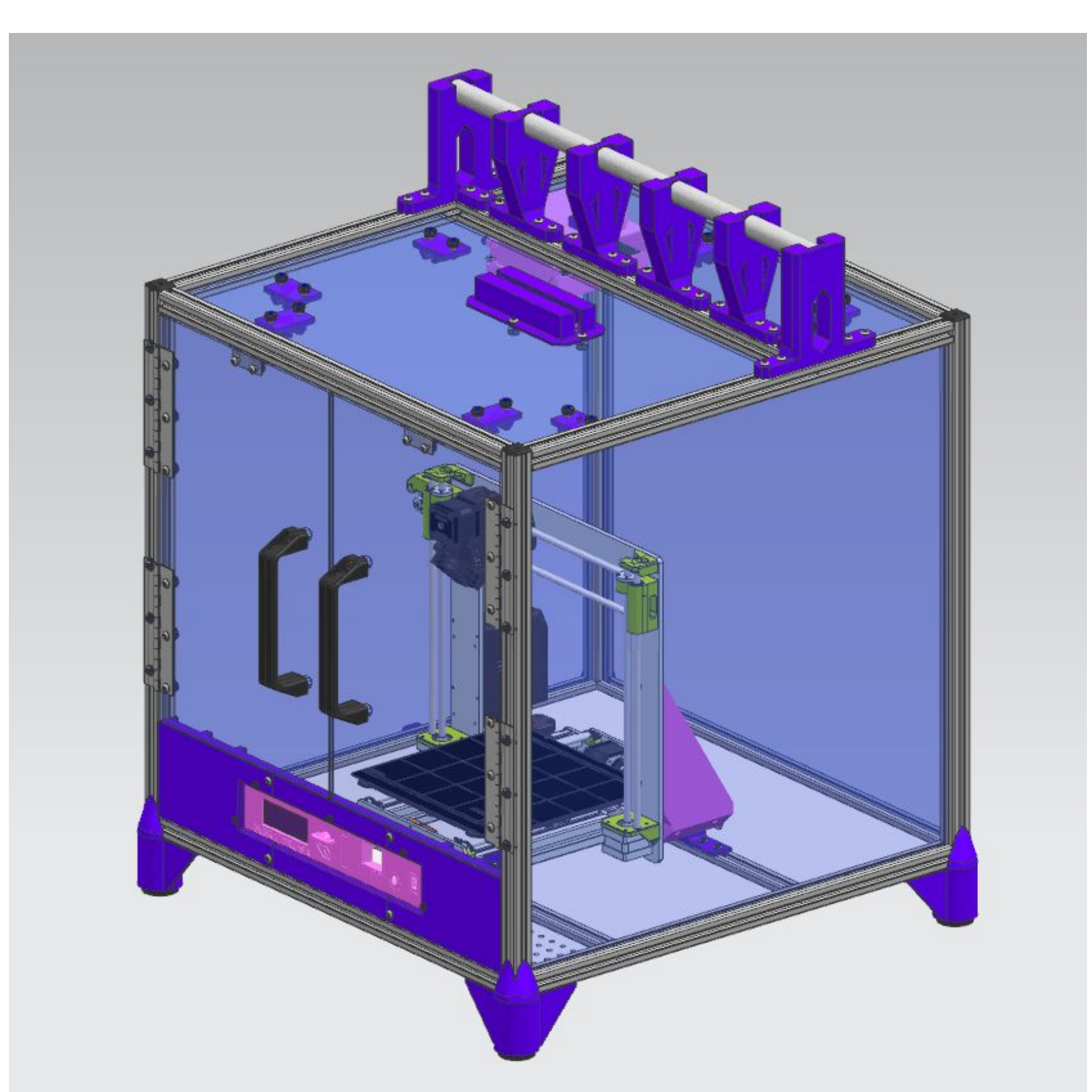

This image displays one of initial designs for the enclosure solution made by Emily Stachowicz.

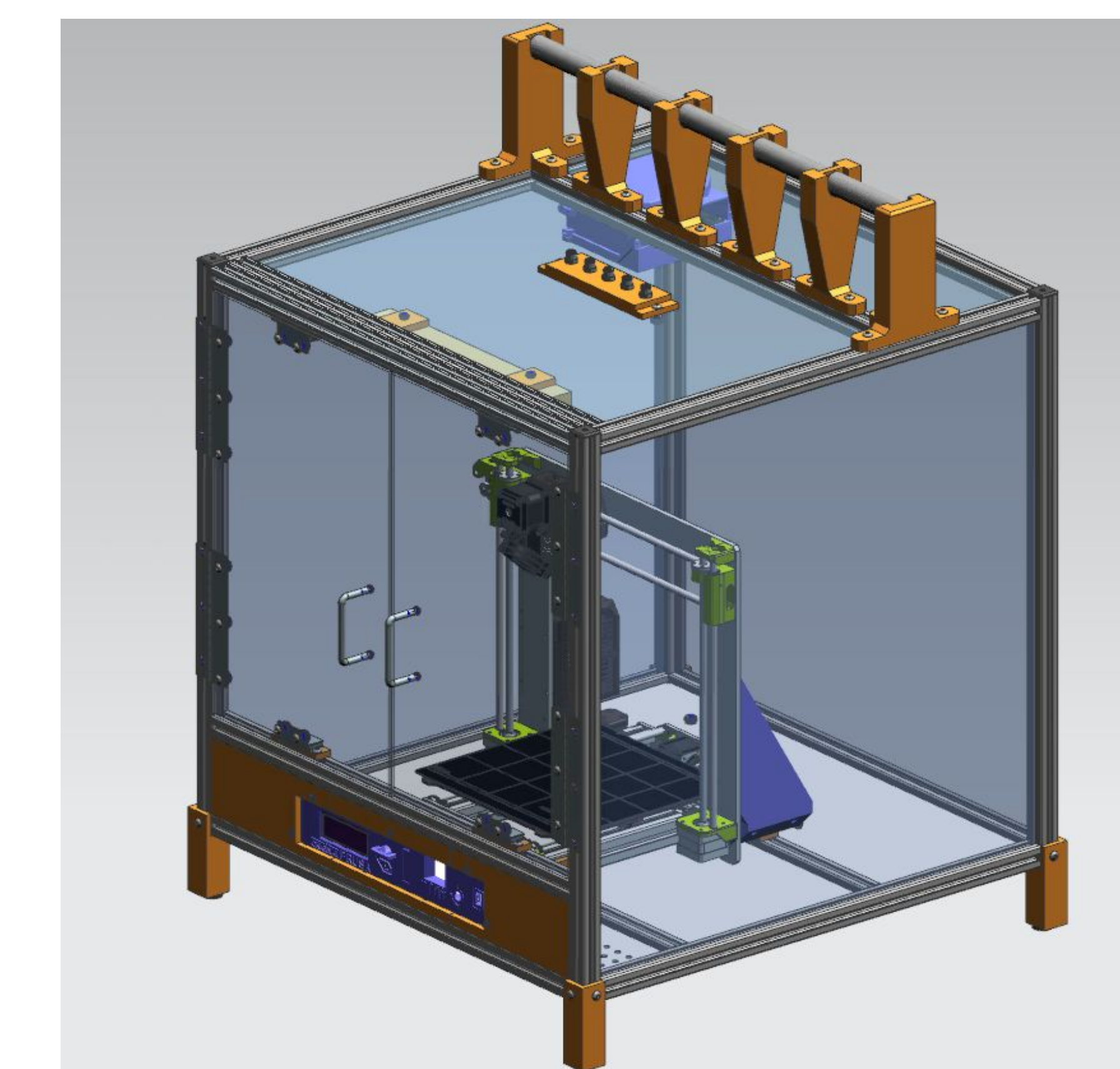

This image displays one of initial designs for the enclosure solution made by Magdalena Sarna. 